\title{
Cancer Therapeutic Effects of Titanium Dioxide Nanoparticles Are Associated with Oxidative Stress and Cytokine Induction
}

\author{
Rina Fujiwara ${ }^{a}$ Yi Luo $^{a} \quad$ Takamitsu Sasaki $^{b}$ Kiyomu Fujii ${ }^{a}$ Hitoshi Ohmoria \\ Hiroki Kuniyasu $^{a}$ \\ ${ }^{a}$ Department of Molecular Pathology, Nara Medical University, Kashihara, and ${ }^{\mathrm{b}}$ Department of Gastroenterological \\ Surgery, Fukuoka University School of Medicine, Fukuoka, Japan
}

\section{Key Words}

Nanoparticles · Oxidants · Cytokines

\begin{abstract}
Nanoparticles (NPs) are considered to influence the inflammatory process; however, the precise mechanism and the significance in tumors are still not clear. In this study, when CT26 and LL2 mouse cancer cells were treated with 6-nm anatase titanium dioxide NPs (TDNPs) without ultraviolet irradiation, oxidative stress and induction of inflammatory cytokines were observed. Oxidative stress was further increased by disease-associated conditions such as high glucose concentrations and hypoxia. Inhaled or orally administered TDNPs generated granulomatous lesions in the lungs and colon of the rodent models tested, with increased oxidative stress and inflammatory cytokines. Oxidative stress and inflammatory cytokines were also found in cancer cells treated with gold or carbon black NPs. Treatment of CT26 cells with 10- to 70-nm rutile TDNPs showed that smaller NPs produced more oxidative stress and inflammatory cytokines than larger ones did. To avoid diffusion of TDNPs and to minimize toxicity, 10-nm TDNPs were suspended in a collagen gel inserted into a subcutaneous tumor in a CT26 mouse. A single TDNP treatment via this
\end{abstract}

method inhibited tumor growth in a size- and dose-dependent manner, and resulted in lower levels of urinary 8-OHdG when compared to systemically administered TDNPs. These findings suggest that TDNPs might be useful for the local treatment of tumors.

(c) 2015 S. Karger AG, Basel

\section{Introduction}

The use of nanotechnology has accelerated in recent years. This technology is associated with advancements in engineering as well as industrial and economic factors [1]. Nanoparticles (NPs) are used ubiquitously in food, medicine, military, construction, cosmetics and sports [2]. Titanium dioxide NPs (TDNPs), in particular, are considered vital in the development of antimicrobiotic applications $[3,4]$. However, there are increasing concerns that nanotechnology may threaten our health [1]. Toxicity has become the focus of extensive research. NPs evoke a natural immune response that is mediated by neutrophils and macrophages [5]. Inhalation of NPs induces persistent respiratory inflammation and plays an important role in respiratory disorders such as chronic obstructive pulmonary disease $[5,6]$. These immunolog-

\section{KARGER 125}

(c) 2015 S. Karger AG, Base

$1015-2008 / 15 / 0826-0243 \$ 39.50 / 0$

E-Mail karger@karger.com

www.karger.com/pat
Hiroki Kuniyasu

Department of Molecular Pathology

Nara Medical University

840 Shijo-cho, Kashihara 634-8521 (Japan)

E-Mail cooninh@zb4.so-net.ne.jp 
ical reactions are thought to be the basis for NP-induced tissue damage.

Anatase TDNPs are common NPs being used for many purposes such as antibacterial effects, sunscreens and cosmetics, making use of their photodynamic properties [4]. The toxic potential of TDNPs is unclear in the presence and absence of ultraviolet (UV) irradiation. We have already examined the effect of UV irradiation-evoked tissue damage in the presence of anatase TDNPs [7]; in those experiments, anatase TDNPs were found to be cytotoxic even in the absence of UV irradiation, suggesting a mechanism based on photodynamism-independent properties.

In this study, we examined the toxic potential and mechanisms of anatase TDNPs. We focused on the physical nature of the NPs, including particle size and chemical composition as well as the production of oxidative stress and cytokines by exposed cells. The possible antitumor application of TDNPs was explored in vivo.

\section{Materials and Methods}

\section{Cells}

The LL2 mouse lung cancer cell line was purchased from the Dainippon Sumitomo Pharma Co., Osaka, Japan. The CT26 mouse colon cancer cell line was a kind gift from Professor I.J. Fidler (MD Anderson Cancer Center, Houston, Tex., USA). Cells were cultured in Dulbecco's modified Eagle's medium supplemented with $10 \%$ fetal bovine serum.

Cell Growth and Apoptosis

Cell growth was assessed using the 3-(4,5-dimethylthiazol2-yl)-2,5-diphenyltetrazolium bromide dye assay, as described previously [8]. Apoptosis was assessed by examining 2,000 cells stained with Hoechst 33342 (Life Technologies, Carlsbad, Calif., USA) using a fluorescent microscope.

\section{Reagents}

TDNPs (10-nm anatase and 10- to $70-\mathrm{nm}$ rutile) were obtained from TEIKA Co., Osaka, Japan. We also purchased gold colloid NPs (10 nm, BBI Solutions, Cardiff, UK), carbon black NPs (15 $\mathrm{nm}$, WAKO Pure Chemicals, Osaka, Japan), glucose (WAKO) and glycated bovine serum albumin (Sigma-Aldrich Chemical Co., St. Louis, Mo., USA).

\section{Ultraviolet Irradiation}

UVA irradiation at 365-nm peak length was performed with a UV lamp (xx-15 BLB, UVP Co., Tokyo, Japan) for $5 \mathrm{~J} / \mathrm{cm}^{2}$. UVB and UVC with $<330 \mathrm{~nm}$ in length were cut with a UV cut filter (UV-33, Toshiba Co., Tokyo, Japan).

\section{Animals}

Four-week-old male BALB/c mice and 5-week-old male Fisher 344 rats were purchased from SLC Japan (Shizuoka, Japan). The animals were maintained according to institutional guidelines ap- proved by the Committee for Animal Experimentation of Nara Medical University and the current regulations and standards of the Japanese Ministry of Health, Labor and Welfare (approval No. 9559).

\section{Animal Models}

To establish a subcutaneous tumor model, CT26 cancer cells $\left(1 \times 10^{7}\right)$ were inoculated into the scapular subcutaneous tissue of $\mathrm{BALB} / \mathrm{c}$ mice. Mice were observed for 4 weeks following inoculation. For evaluating the effect of anatase or rutile TDNPs, different sizes, i.e. $200 \mu \mathrm{g}$ in $200 \mu \mathrm{l}$ of phosphate-buffered saline (PBS), were injected into the CT26 subcutaneous tumor. For the NP aspiration model, TDNPs suspended in $200 \mu \mathrm{l}$ of PBS were aspirated into the nose of Fischer 344 rats under anesthesia. For the NP oral model, TDNPs suspended in $200 \mu \mathrm{l}$ PBS were administrated to BALB/c mice intragastrically. The toxicity of the different forms of TDNPs $(200 \mu \mathrm{g})$ to BALB/c mice was determined following subcutaneous or intraperitoneal injection.

\section{Titanium Gel Plug}

Rutile TDNPs $(200 \mu \mathrm{g})$ were mixed with melted $1.5 \%$ agar-PBS at $37^{\circ} \mathrm{C}$. The suspension was cooled in the lumen of an 18-gauge needle. The needle containing the titanium gel plug was inserted into the tumor and the plug was then expelled. For evaluation of toxicity of the titanium gel plug, one was inserted into the subcutaneous tissue of $\mathrm{BALB} / \mathrm{c}$ mice without tumors.

\section{Tissue Titanium}

The subcutaneous tumor $(1 \mathrm{~g})$ was digested with proteinase $\mathrm{K}$ for $2 \mathrm{~h}$ at $45^{\circ} \mathrm{C}$, followed by treatment with $65 \%$ nitric acid overnight at $80^{\circ} \mathrm{C}$. The suspension was analyzed using a flameless atomic absorption spectrometer (AAS) to measure the titanium concentration $(\lambda=226.8 \mathrm{~nm})$, using an AAS titanium standard (Sigma-Aldrich).

\section{Reverse Transcription-Polymerase Chain Reaction}

Total RNA ( $1 \mu \mathrm{g})$ was synthesized using the ReverTra Ace quantitative PCR (qPCR) RT kit (Toyobo, Osaka, Japan). The primer sets for amplification were listed below. Mouse TNFA (accession No. D84199.2): upper 5' -AAG ATG GAG GAA GGG CAG TT-3' ${ }^{\prime}$, lower $5^{\prime}$-GAT CCT GGA GGG GAA GAG AC-3'; mouse $I L-1 B$ (accession No. NM_008361.3): upper $5^{\prime}$-GCC CAT CCT CTG TGA CTC AT3', lower 5'-AGG CCA CAG GTA TTT TGT CG-3'; mouse CXCL2 (accession No. NM_009140.2): upper $5^{\prime}$-AAG TTT GCC TTG ACC CTG AA-3', lower 5'-AGG CAC ATC AGG TAC GAT CC-3'; mouse HMGB1 (accession No. NM_010439.3): upper 5'-ACA GAG CGG AGA GAG TGA GG-3', lower $5^{\prime}$-GGG TGC TTC TTC TTG TGC TC-3'; mouse ACTB (accession No. NM_007393.4): upper 5' AGC CAT GTA CGT AGC CAT CC-3', lower $5^{\prime}$-CTC TCA GCT GTG GTG GTG AA-3'. The primers were synthesized by Sigma Genosys (Ishikari, Japan). The PCR conditions were set according to the provider's instructions. PCR products were electrophoresed on a $2 \%$ agarose gel and visualized using ethidium bromide.

\section{Enzyme-Linked Immunosorbent Assay}

ELISA kits were used to measure concentrations of 4-hydroxynonenal (4-HNE; Cusabio Biotech Co. Ltd., Wilmington, Del., USA), 8-hydroxy-2'-deoxyguanosine (8-OHdG; Japan Institute for the Control of Aging, Shizuoka, Japan) and high mobility group box-1 (HMGB1; Shino-test Co., Tokyo, Japan), according to the manufacturers' instructions. 
Fig. 1. Oxidative stress generated from anatase TDNPs. a, b Effect of anatase TDNPs on CT26 cell number with $(\mathrm{UV}+)$ and without (UV-) UVB irradiation. c Cytotoxicity of anatase TDNPs in CT26 cells without UVB as a function of concentration and time. d Time-dependent apoptosis induced by anatase TDNP without UVB in LL2 and CCT26 cells. 4-HNE (e) and 8 -OHdG (g) were measured in CT26 cells by increasing concentrations of anatase TDNPs with and without UVB. 4-HNE (f) and 8-OHdG (h) generated by anatase TDNPs $(200 \mu \mathrm{g} / \mathrm{ml})$ with and without UVB. Each treatment was continued for $24 \mathrm{~h}$. Data are expressed as means \pm SD from 3 independent trials. Acid $=$ pH 6.5 medium adjusted by citrate; $\mathrm{HG}=$ high glucose concentration $(400 \mathrm{mg} / \mathrm{ml})$; HT = high temperature $\left(40^{\circ} \mathrm{C}\right) ;$ Hypo = hypoxia $\left(5 \% \mathrm{O}_{2}\right)$; $\mathrm{UV}=\mathrm{UVB}$
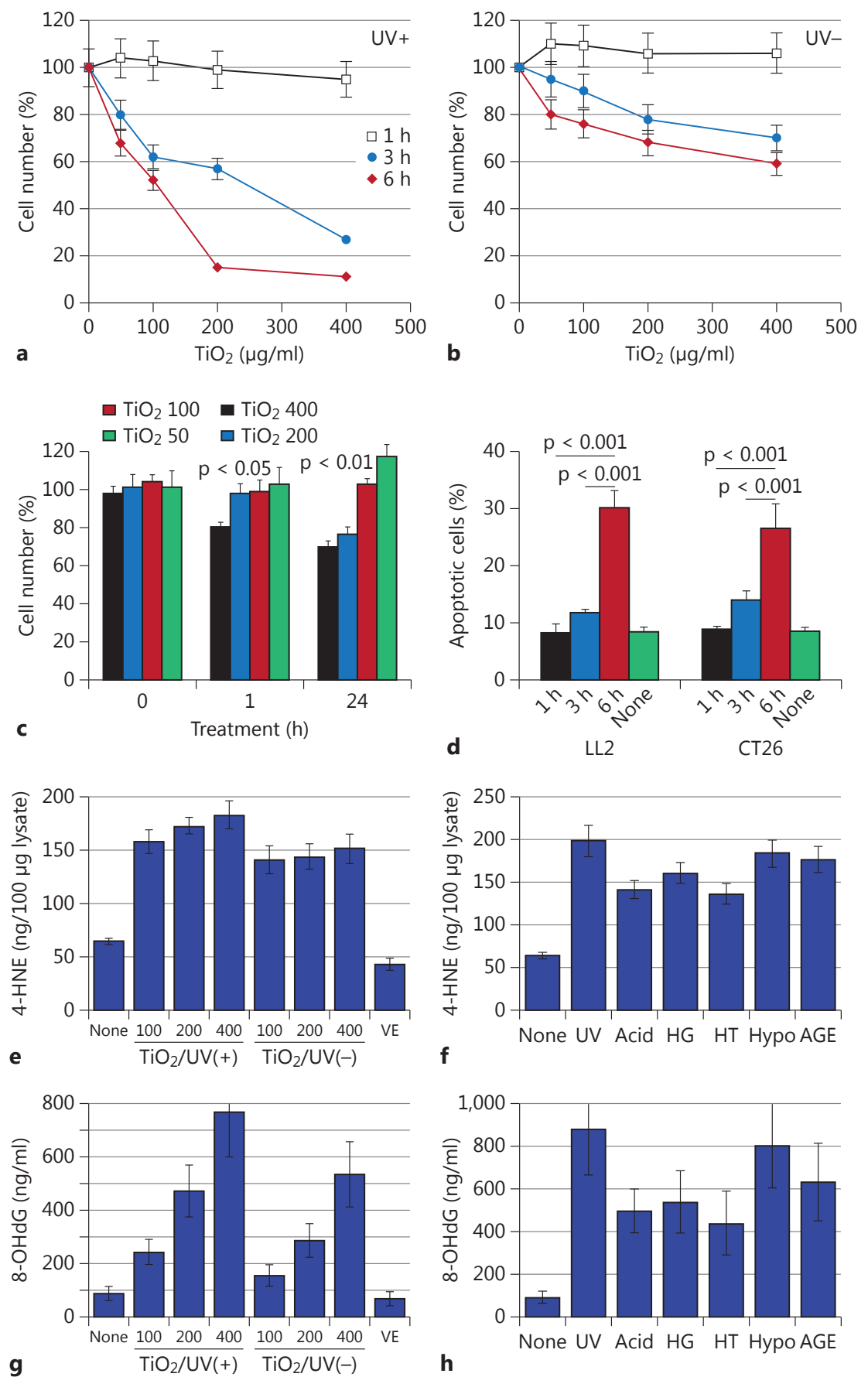

h

previously reported [9]. TUNEL assay was performed using the in situ cell death detection kit, POD (Roche Diagnostics, Indianapolis, Ind., USA). The percent frequency of Ki-67- or TUNEL-positive cells was calculated from the ratio of positive nuclei to 500 examined nuclei. 

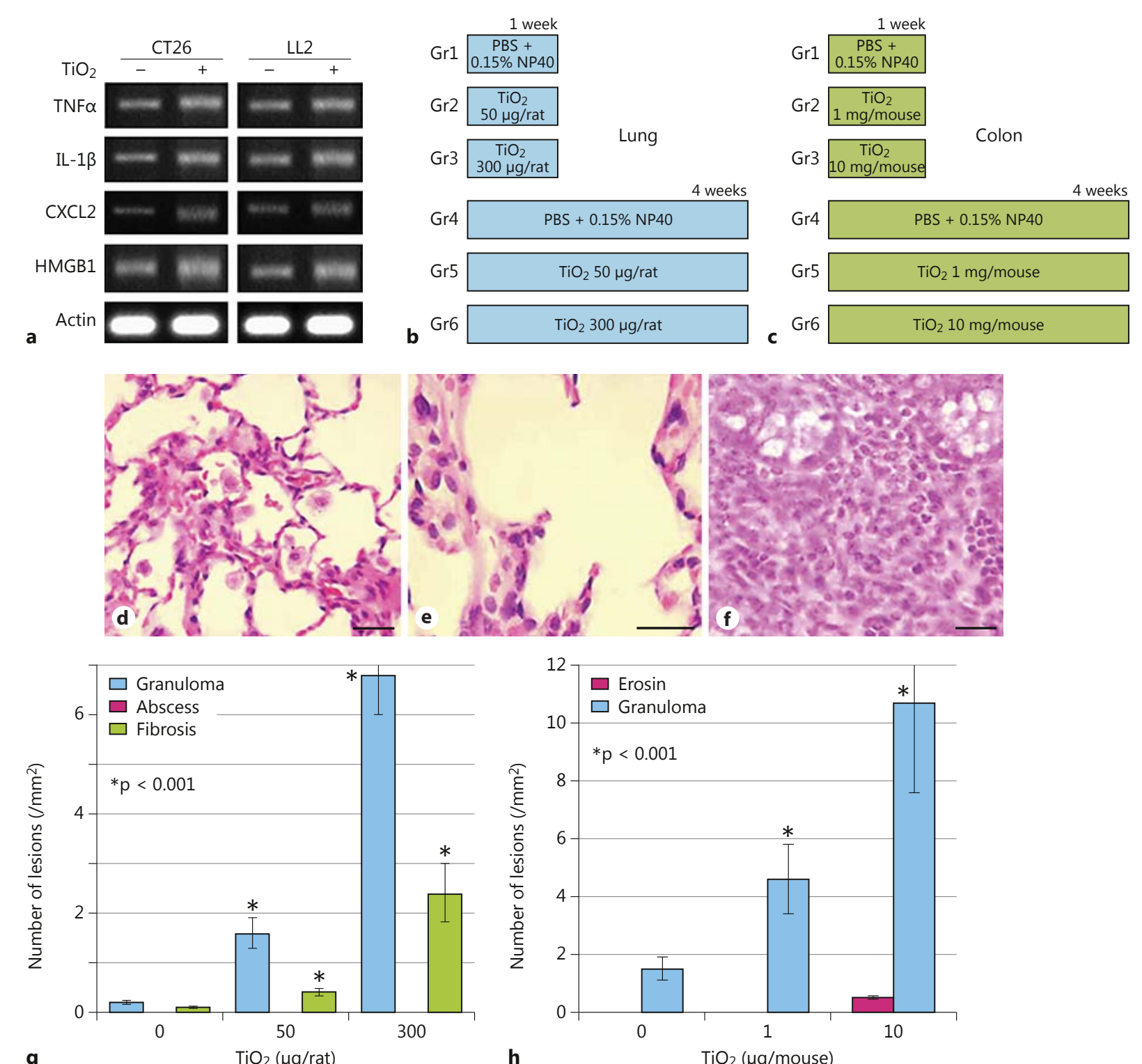

$\mathrm{TiO}_{2}(\mu \mathrm{g} / \mathrm{rat})$

h

$\mathrm{TiO}_{2}$ ( $\mu \mathrm{g} /$ mouse)
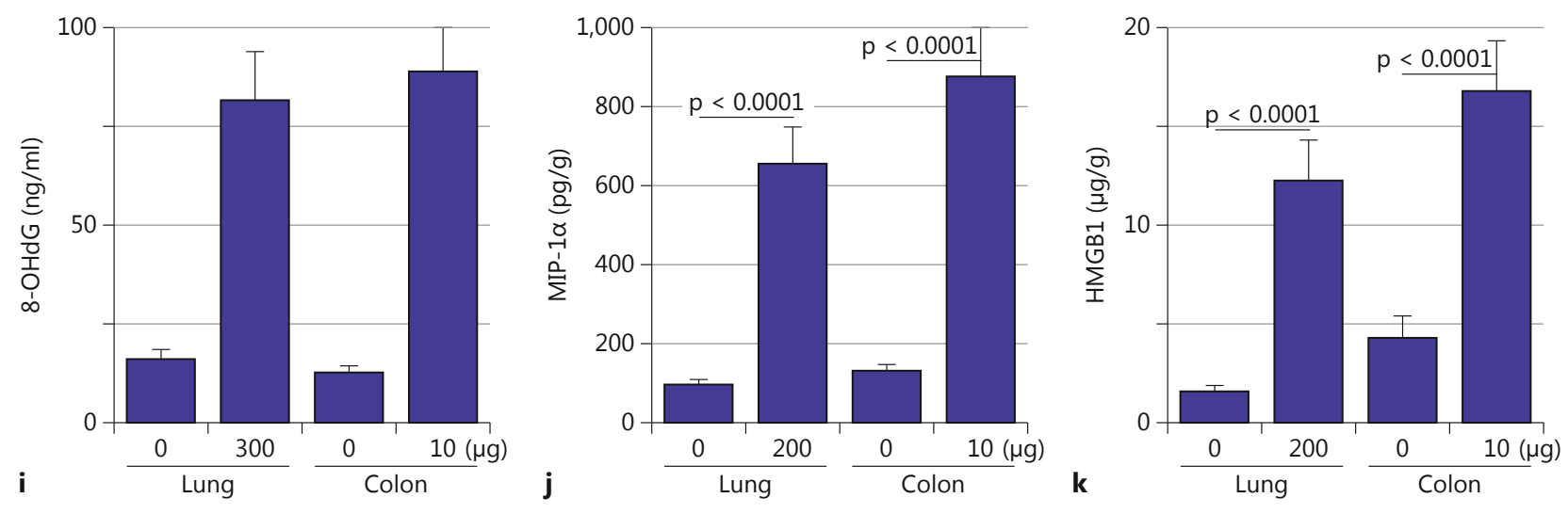

(For legend see next page.) 
Statistical Analysis

Statistical significance was calculated by using the two-tailed Fisher exact test, the $\chi^{2}$ test and the unpaired Mann-Whitney U test with InStat software (Graphpad, Los Angeles, Calif., USA). Statistical significance was defined as a two-sided $p$ value of $<0.05$.

\section{Results}

\section{Cytotoxicity of Anatase TDNPs}

The cytotoxicity of anatase TDNPs was assessed with or without UVB irradiation $\left(1.5 \mathrm{~J} / \mathrm{cm}^{2}\right)$ in CT26 cells (fig. 1a, b). Both treatments showed a $\mathrm{TiO}_{2}$ concentration-dependent cytotoxicity. However, UVB treatment increased cytotoxicity 2 -fold. The cytotoxic effect of anatase TDNPs was confirmed in LL2 cells (fig. 1c) and was found to involve the induction of apoptosis in both LL2 and CT26 cells (fig. 1d).

\section{Induction of Oxidative Stress by Anatase TDNPs}

The oxidative stress generated by anatase TDNPs was assessed by measuring 4-HNE and 8-OHdG (fig. 1e, g). 4-HNE levels in CT26 cells treated with various concentrations of anatase TDNPs without UVB were about $80 \%$ of those in cells treated with TDNPs with UVB. 8-OHdG levels in CT26 cells treated with anatase TDNPs without UVB were about $60 \%$ of those in cells treated with anatase TDNPs and UVB. Various physiological conditions were examined for their effect on the oxidative stress induced by treatment with anatase TDNPs (fig. 1f, h). Acidic condition ( $\mathrm{pH}$ 6.5), hyperglycemia (400 $\mathrm{mg} / \mathrm{dl})$, hyperthermia $\left(38^{\circ} \mathrm{C}\right)$, hypoxia $\left(5 \% \mathrm{O}_{2}\right)$ and advanced glycation end products (AGE, $100 \mu \mathrm{g} / \mathrm{ml}$ glycated bovine serum albu$\mathrm{min}$ ) increased the levels of both 4-HNE and 8-OHdG in CT26 cells treated with anatase TDNPs.

\section{Induction of Cytokines by TDNPs in Cancer Cells}

The expression of TNF $\alpha$, IL-1 $\beta$, CXCL 2 and HMGB1 mRNA was examined by RT-PCR in TDNP-treated

Fig. 2. Tissue damage induced by anatase TDNPs. a mRNA expression of cytokines in CT26 and LL2 mouse cancer cells treated with anatase TDNPs $(200 \mu \mathrm{g} / \mathrm{ml})$ without UV irradiation. b, c Protocols used for the F344 rat aspiration and BALB/c mouse oral dosing models using anatase TDNPs ( $\mathrm{n}=5$ for each group). $\mathbf{d}$, e Rat lung sections. Alveoli exhibited granulomatous changes and mild fibrosis. HE. f Mouse colon mucosa showing granulomatous changes. HE. g, $\mathbf{h}$ Frequency of histological alterations in rat lung and mouse colon. $\mathbf{i}-\mathbf{k}$ Levels of 8-OHdG, MIP-1 $\alpha$ and HMGB1 in rat lungs and mouse colons after treatment with TDNPs. Data are expressed as means \pm SD from 5 animals or 3 independent trials.
CT26 and LL2 cells (fig. 2a). Expression of these cytokines was upregulated by TDNP treatment in both cancer cell lines.

\section{Effect of TDNPs on Tissue Reactions in the Lung and Colon}

To examine the effect of TDNPs on tissues, the lungs and colon of rodents were exposed according to protocol (fig. 2b, c). In rat lungs and mouse colon, granulomas were observed in a dose-dependent manner (fig. 2d-h). In these tissues, oxidative stress, detected by $8-\mathrm{OHdG}$ and the macrophage-activating cytokines MIP-1 $\alpha$ and HMGB1, were markedly increased (fig. 2i-k).

\section{Induction of Oxidative Stress by Other NPs}

To examine the hypothesis that the induction of oxidative stress is a common feature of NPs, rutile TDNPs, gold NPs and carbon black NPs were tested. The cytotoxicity of all 3 types of NPs was similar in CT26 cells (fig. 3a). The levels of 4-HNE and 8-OHdG were increased in cells treated with the 3 types of NPs (fig. 3b, c) with or without hyperglycemic or hypoxic conditions (fig. $3 \mathrm{~d}, \mathrm{e}$ ). The mRNA expression of the macrophage-activating cytokines HMGB1 and MIP-1 1 was also upregulated by these NPs (fig. 3f).

\section{Effect of Particle Size on the Activities of NPs}

The effect of rutile TDNP particle size on the induction of oxidative stress, the expression of cytokines and tissue damage was determined in CT26 cells. The smaller the NPs, the higher were the levels of $4-\mathrm{HNE}$ and $8-\mathrm{OHdG}$ (fig. $3 \mathrm{~g}, \mathrm{~h}$ ). Using the protocol shown in figure $2 \mathrm{~b}, 5 \mathrm{dif}-$ ferent sizes of rutile TDNPs were administered into the lungs of rats. Lung weight was increased and the number of granulomas was more pronounced in rats treated with smaller NPs (fig. 3i-1). Smaller NPs also more actively induced HMGB1 expression and the formation of 8-OHdG (fig. $3 \mathrm{~m}, \mathrm{n}$ ).

\section{TDNP as a Local Tumor Treatment}

The anti-tumor effects of rutile TDNPs in vitro and of a gel plug containing TDNPs that was embedded into a tumor in vivo, were examined. As shown in figure $4 a-c$, smaller TDNPs showed a more pronounced inhibition of CT26 cell growth as well as relatively more oxidative stress. Six- and 10-nm NPs inhibited cell growth by $60 \%$ and caused high levels of 4 -HNE and 8-OHdG.

In vivo treatment with a TDNP gel plug induced tumor cell death in the tumor (fig. 4d). In degraded tumor 

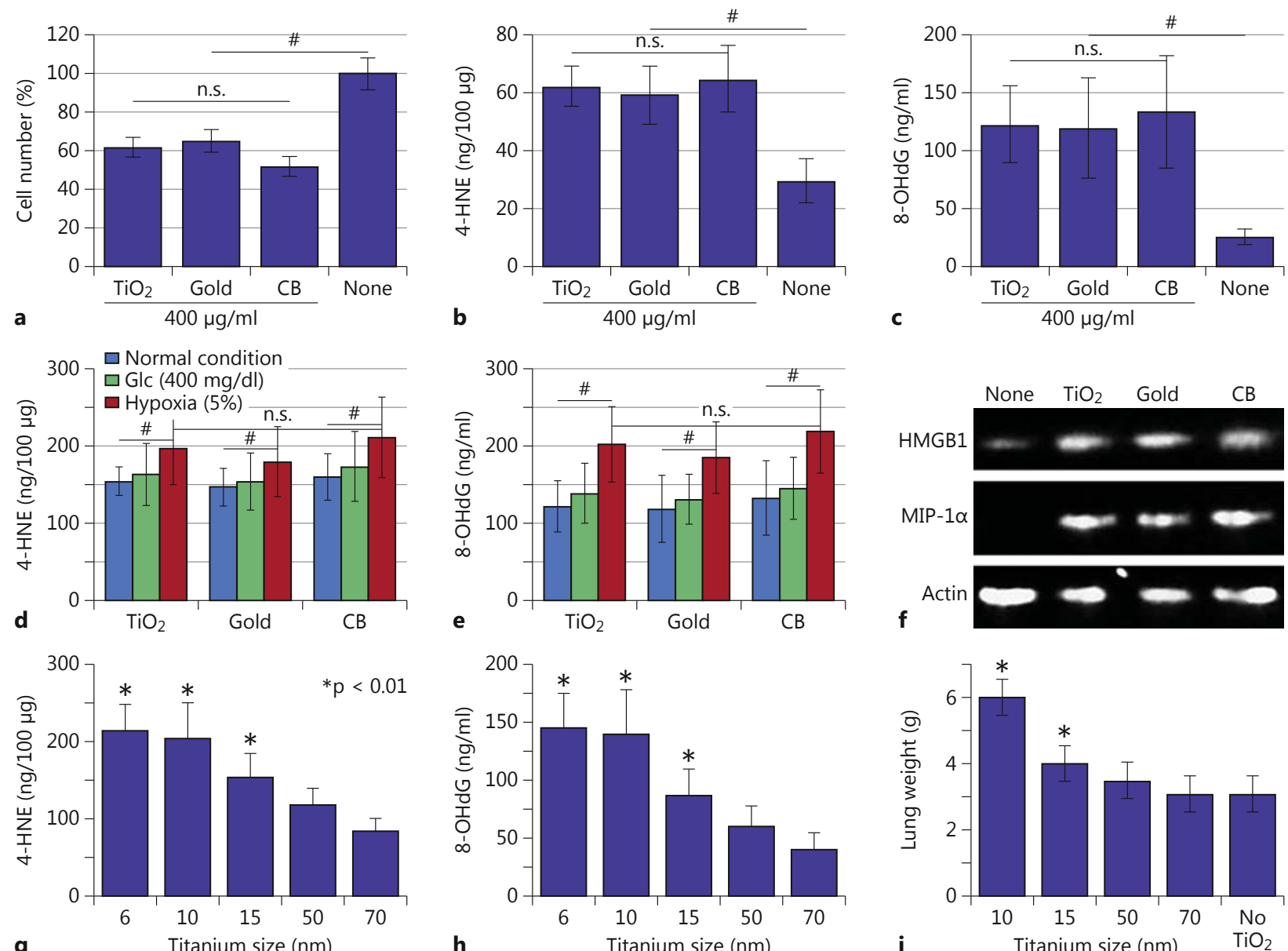

g

Titanium size (nm)

h
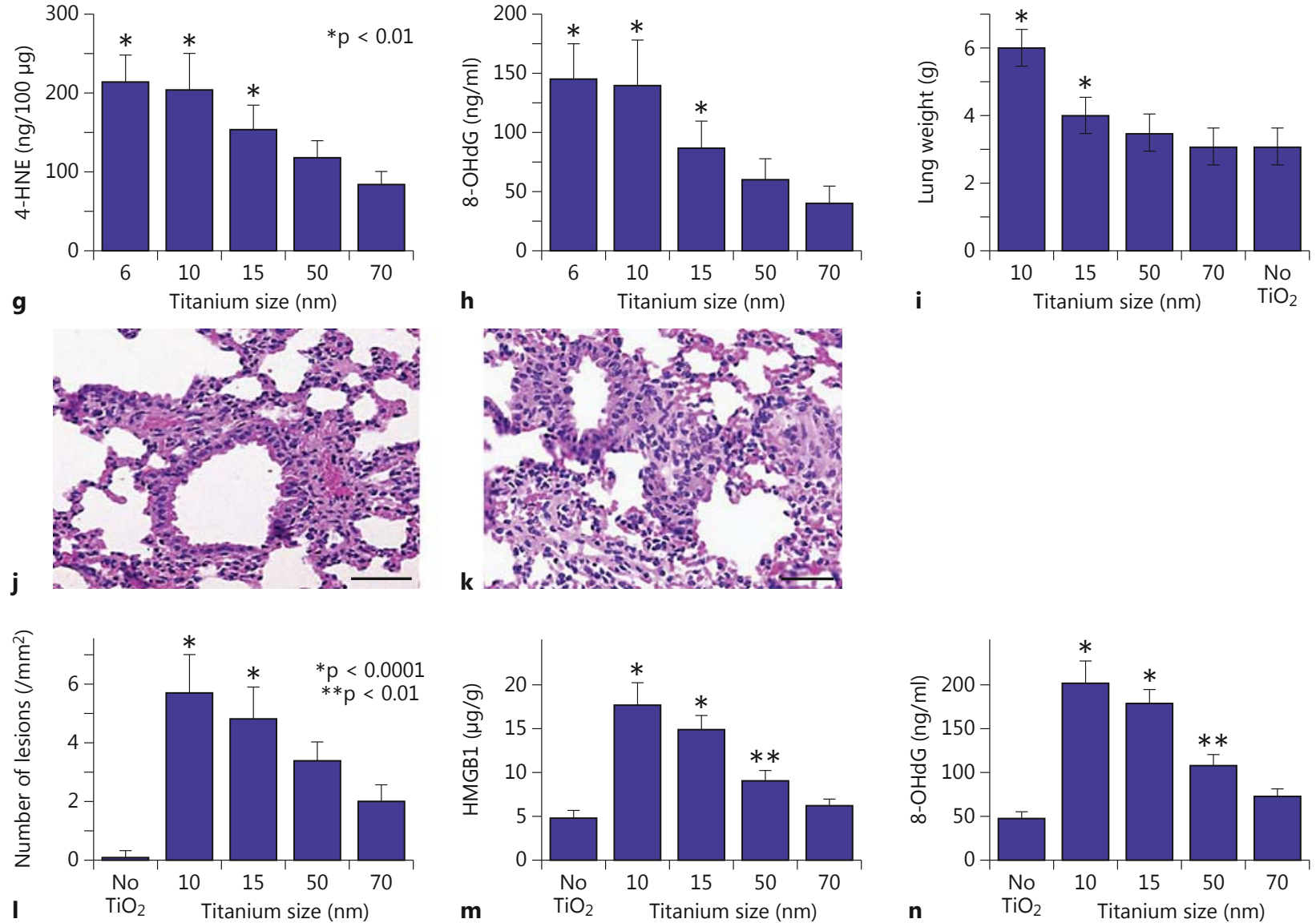

3

(For legend see next page.)

Fujiwara/Luo/Sasaki/Fujii/Ohmori/ Kuniyasu 
cells, TDNP aggregations were found (fig. $4 \mathrm{~d}$ inset). Titanium contents in the tumors were higher in $10-\mathrm{nm}$ TDNP-treated tumors than those in 70-nm TDNP-treated tumors (fig. 4e). In 10-nm TDNP-treated tumors, TUNEL-positive apoptotic cells were increased and Ki67-positive proliferating cells were decreased in comparison to those in tumors treated with gel alone (fig. 4f). Intratumoral insertion of the TDNP gel plug inhibited tumor growth in an NP size-, dose- and time-dependent manner (fig. $4 \mathrm{~g}, \mathrm{~h}$ ).

The local effect of TDNPs in the nontumoral tissues was evaluated by insertion of a TDNP gel plug into the subcutaneous tissue of the mouse with no tumor (fig. 4i). Thick fibrosis occurred in the insertion site in the subcutaneous tissue; however, no alteration was observed at the fascia and skeletal muscle. The systemic effect of TDNPs was evaluated by measuring urinary excretion of $8-\mathrm{OHdG}$ as an index of general oxidative toxicity. Mice without a tumor were treated with rutile TDNPs or TDNP gel (10 $\mathrm{nm}, 100 \mu \mathrm{g})$. Subcutaneous or intraperitoneal administration of TDNPs suspended in PBS resulted in high concentrations of 8-OHdG in the urine. TDNP gel, administered intraperitoneally, melted and yielded the same levels of urine $8-\mathrm{OHdG}$ (fig. 4j). In contrast, TDNP gel inserted into the subcutaneous tissue was thick with fibrosis and provided lower levels of urinary 8-OHdG than in the other groups (fig. $4 \mathrm{j}$ ). These findings suggest that intratumoral administration of a TDNP gel was effective for inhibiting the growth of cancer cells with low toxicity to the host.

Fig. 3. Effect of NP composition and size on indices of toxicity and oxidative stress. a-c Changes in cell number, 4-HNE and 8-OHdG in CT26 cells treated with $400 \mu \mathrm{g} / \mathrm{ml}$ rutile TDNPs $(10 \mathrm{~nm})$, gold $(10 \mathrm{~nm})$ or carbon black (CB, $15 \mathrm{~nm})$. d, e Effect of high glucose concentration (Glc, $400 \mathrm{mg} / \mathrm{ml})$ and hypoxia $\left(5 \% \mathrm{O}_{2}\right)$ on 4 -HNE and $8-O H d G$ in CT26 cells treated with $200 \mu \mathrm{g} / \mathrm{ml}$ of TDNPs (10 $\mathrm{nm})$, gold $(10 \mathrm{~nm})$ or CB $(15 \mathrm{~nm})$. f Expression of MIP-1 1 and HMGB1 mRNA in CT26 cells treated with $200 \mu \mathrm{g} / \mathrm{ml} \mathrm{TiO}{ }_{2}(10$ $\mathrm{nm})$, gold $(10 \mathrm{~nm})$ or CB $(15 \mathrm{~nm}) . \mathbf{g}, \mathbf{h}$ Effect of treating CT26 cells with different sizes of rutile TDNPs $(200 \mu \mathrm{g} / \mathrm{ml})$ on 4 -HNE and 8-OHdG levels. i Effect of different sizes of rutile TDNPs on lung damage in the rat aspiration model ( $n=5$ for each group). $\mathbf{j}, \mathbf{k}$ Rat alveoli 4 weeks after treatment with TDNPs. HE. $\mathbf{j}$ In rats treated with 70-nm TDNPs, minimal granulomatous changes are evident. k In rats treated with $10-\mathrm{nm}$ TDNPs, marked granulomatous changes and thickening of the alveolar wall are evident. Number of granulomatous lesions (I), concentrations of HMGB1 (m) and concentrations of 8-OHdG (n) in the rat lungs following treatment with different sizes of TDNPs. Data are expressed as means \pm SD from 5 animals or 3 independent trials. ${ }^{*} \mathrm{p}<0.05$.

\section{Discussion}

In this study, the toxicity of anatase and rutile TDNPs was determined with and without concomitant UV irradiation. TDNPs caused oxidative stress and induced the production of inflammatory cytokines in vivo. These effects were enhanced by UV irradiation. Smaller NPs generated more pronounced levels of oxidative stress and cytokines than did larger NPs. NPs caused granulomatous inflammation in the lung and intestine. Importantly, TDNPs damaged tumor tissue following intratumoral administration. TDNP administered into tumors formed micrometer-level aggregation, as reported previously [10], and stayed in degraded tumor tissue.

We found that treating CT26 cells with TDNPs produced oxidative stress without UV. This observation was consistent with other reports [11-13]. We also confirmed that gold and carbon black NPs induced oxidative stress. These results indicated that oxidative stress is a common feature of treating cells with NPs.

The mechanism by which NPs generate oxidants in an acellular system is reported to be based upon pro-oxidative functional groups at the NP surface and redox cycling of transition metals $[14,15]$. We used NPs devoid of transition metals to evaluate acellular oxidant production. With such NPs, surface and size might be pivotal factors for generating oxidants. A size effect was reported for carbon black NPs [12]. In addition, the surface area of carbon black NPs correlated with the generation of oxidants.

Oxidative stress produced in response to TDNP administration was enhanced by high glucose concentrations, acidic $\mathrm{pH}$, hypoxia, a high temperature and the presence of AGE. This enhancement might be associated with the direct effects of NPs on cells to produce free radicals and other oxidants. In addition, NP-derived oxidative stress in vivo may involve mitochondria or $\operatorname{NAD}(\mathrm{P}) \mathrm{H}$ oxidase $[13,14]$. The conditions that enhance TDNP-mediated oxidative stress may affect the redox status of mitochondrial oxidative phosphorylation. Such conditions are present in patients with diabetes and ischemia and NPs may be more toxic to such individuals.

Paradoxically, NPs might play a role in scavenging oxidants generated by hyperglycemia in an acellular system $[16,17]$. In hypoxia, NPs derived from fullerene showed antioxidant effects in the wound-healing process [18]. These findings suggest that the particle surface might provide NPs with antioxidant properties, although our current data suggest that NPs are more likely to adversely affect these disease-associated conditions. It is noteworthy that cancer tissues are commonly hypoxic and 


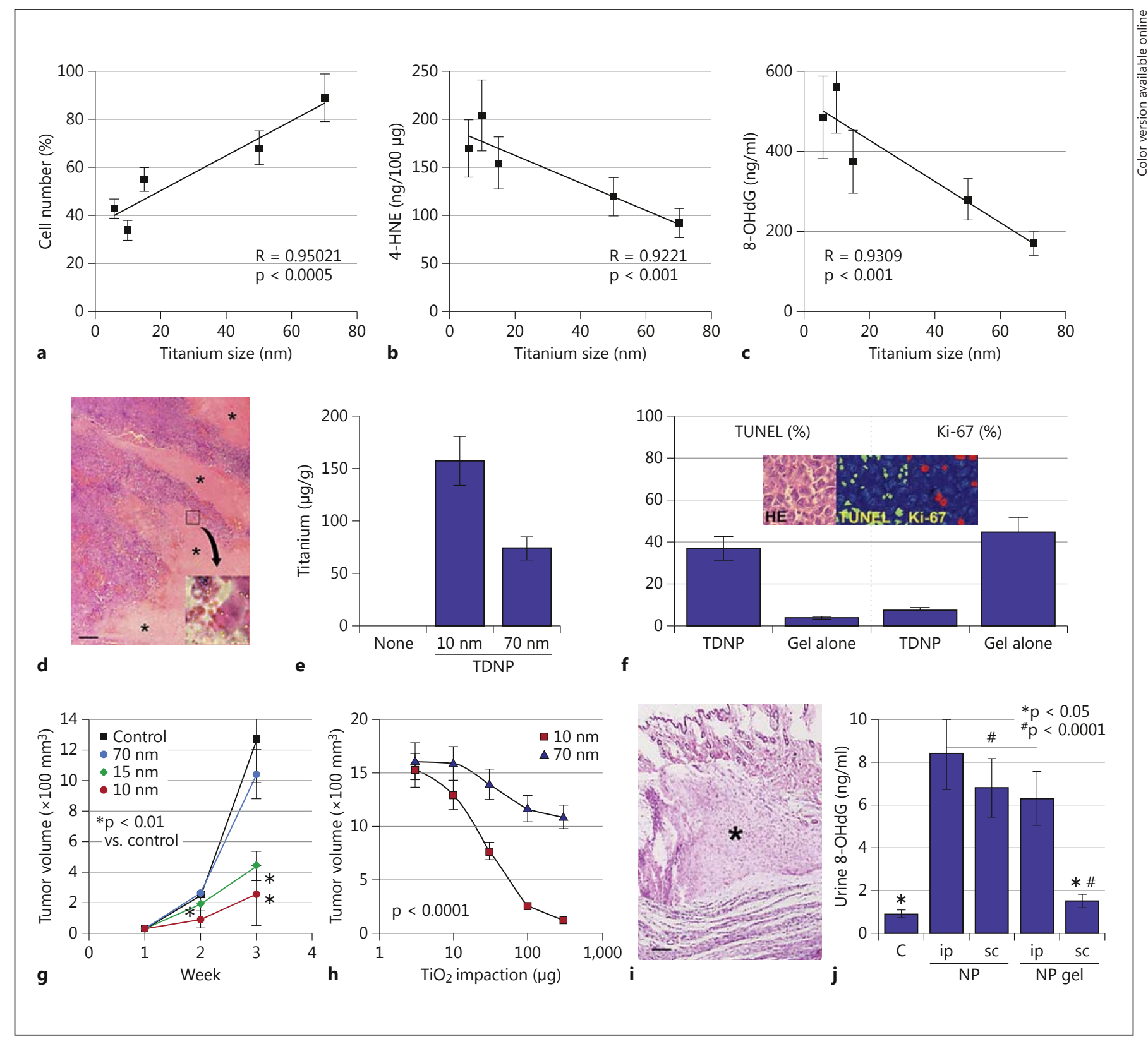

Fig. 4. Effects of rutile TDNPs on cancer cells in vivo and in vitro. a-c Effects of treating CT26 cells for $48 \mathrm{~h}$ with different sizes of rutile TDNPs $(200 \mu \mathrm{g} / \mathrm{ml})$ on cell number, 4 -HNE and $8-\mathrm{OHdG}$. d-f Effect of TDNP gel plug on tumor. d Massive cell death (asterisk) in the CT26 tumor treated with 10-nm TDNPs. Bar: $100 \mu \mathrm{m}$. Inset Aggregation of TDNPs in degraded tumor cells. e Titanium contents in the tumors were measured by AAS. $\mathbf{f}$ TUNEL-positive apoptotic cells and $\mathrm{Ki}-67$-positive proliferating cells in the CT26 tumor treated with 10-nm TDNP or gel alone. Inset Representatives in the tumor treated with 10-nm TDNP. g Effect of different sizes of rutile TDNPs in a gel on the in vivo growth of CT26 subcutaneous tumors ( $\mathrm{n}=5$ mice in each group). $\mathbf{h}$ Dose-dependent effects of 10- and 70-nm rutile TDNP gel on the in vivo growth of CT26 subcutaneous tumors ( $\mathrm{n}=5$ mice in each group). $\mathbf{i}$ Effect of the TDNP gel plug on nontumoral tissue: it was inserted into the subcutaneous tissue of tumor-negative mice. Subcutaneous fibrosis (asterisk). $\mathbf{j}$ Effect of intraperitoneal and subcutaneous rutile TDNP gel on urinary 8-OHdG after 1 week. Data are expressed as means \pm SD of 4 mice per group. SD from 5 animals or 3 independent trials. 
acidic [19]. This may enhance the oxidative stress produced by NPs, and suggests that NPs may be an effective anticancer treatment.

In our in vitro and in vivo experiments, inflammatory cytokines, such as MIP-1 $\alpha$ and HMGB1, were produced by cancer cells and noncancerous tissues. These cytokines might enhance oxidative stress and activate granulomatous macrophages $[20,21]$. This is recognized as the third mechanism of NP-associated oxidative stress [14]. HMGB1, a late-phase inflammatory cytokine, is secreted from macrophages activated with IL- $1 \beta$ or TNF $\alpha$ and promotes their secretion [21]. This vicious cycle between inflammatory cytokines and oxidative stress plays an important role in the cellular and tissue damage caused by NPs.

NPs generally induce oxidative stress, activate caspase-3 and induce apoptosis [22]. Thus, nonselective diffusion of NPs has the potential to evoke damage in noncancerous tissues. To avoid this potential toxicity, rutile TDNPs were intratumorally administered in a gel plug. Our data show that TDNPs administered in this manner induced apoptosis, suppressing the growth of remnant cancer cells otherwise stimulated by HMGB1 released from dead cells [23].

Overall, our data suggest that the local use of TDNPs is an effective cancer treatment. A single, localized dose of TDNPs might produce oxidative stress and inhibit cancer growth for a prolonged period of time with minimal side effects. In further study, examination of the antitumor effect and the side effects of long-term administration of TDNP are needed.

\section{Acknowledgments}

This work was supported in part by grants-in-aid for Scientific Research from the Japan Society for the Promotion of Science (11017371) and the Ministry of Health, Labor and Welfare, Japan (11103430).

\section{References}

1 Singh N, Manshian B, Jenkins GJ, Griffiths SM, Williams PM, Maffeis TG, Wright CJ, Doak SH: Nanogenotoxicology: the DNA damaging potential of engineered nanomaterials. Biomaterials 2009;30:3891-3914.

2 Kwon JY, Koedrith P, Seo YR: Current investigations into the genotoxicity of zinc oxide and silica nanoparticles in mammalian models in vitro and in vivo: carcinogenic/genotoxic potential, relevant mechanisms and biomarkers, artifacts, and limitations. Int J Nanomed 2014; 9(suppl 2):271-286.

3 Pelgrift RY, Friedman AJ: Nanotechnology as a therapeutic tool to combat microbial resistance. Adv Drug Deliv Rev 2013;65:1803-1815.

4 Yin ZF, Wu L, Yang HG, Su YH: Recent progress in biomedical applications of titanium dioxide. Phys Chem Chem Phys 2013;15:48444858.

5 Morimoto Y, Izumi H, Kuroda E: Significance of persistent inflammation in respiratory disorders induced by nanoparticles. J Immunol Res 2014;2014:962871.

6 Li JJ, Muralikrishnan S, Ng CT, Yung LY, Bay BH: Nanoparticle-induced pulmonary toxicity. Exp Biol Med 2010;235:1025-1033.

7 Chihara Y, Fujimoto K, Kondo H, Moriwaka Y, Sasahira T, Hirao Y, Kuniyasu H: Anti-tumor effects of liposome-encapsulated titanium dioxide in nude mice. Pathobiology 2007;74:353358.

8 Kuniyasu H, Yano S, Sasaki T, Sasahira T, Sone $\mathrm{S}$, Ohmori H: Colon cancer cell-derived high mobility group $1 /$ amphoterin induces growth inhibition and apoptosis in macrophages. Am J Pathol 2005;166:751-760.
9 Kuniyasu H, Luo Y, Fujii K, Sasahira T, Moriwaka Y, Tatsumoto N, Sasaki T, Yamashita Y, Ohmori H: CD10 enhances metastasis of colorectal cancer by abrogating the anti-tumoural effect of methionine-enkephalin in the liver. Gut 2010;59:348-356.

10 Chowdhury I, Walker SL, Mylon SE: Aggregate morphology of nano- $\mathrm{TiO}_{2}$ : role of primary particle size, solution chemistry, and organic matter. Environ Sci Process Impacts 2013;15:275282.

11 Sarkar A, Ghosh M, Sil PC: Nanotoxicity: oxidative stress mediated toxicity of metal and metal oxide nanoparticles. J Nanosci Nanotechnol 2014;14:730-743.

12 Koike E, Kobayashi T: Chemical and biological oxidative effects of carbon black nanoparticles. Chemosphere 2006;65:946-951.

13 Knaapen AM, Borm PJ, Albrecht C, Schins RP: Inhaled particles and lung cancer. Part A. Mechanisms. Int J Cancer 2004;109:799-809.

14 Manke A, Wang L, Rojanasakul Y: Mechanisms of nanoparticle-induced oxidative stress and toxicity. Biomed Res Int 2013;2013:942916.

15 Schins RP: Mechanisms of genotoxicity of particles and fibers. Inhal Toxicol 2002;14:57-78.

16 Ghaznavi H, Najafi R, Mehrzadi S, Hosseini A, Tekyemaroof N, Shakeri-Zadeh A, Rezayat M, Sharifi AM: The neuro-protective effects of cerium and yttrium oxide nanoparticles on high glucose-induced oxidative stress and apoptosis in undifferentiated PC12 cells. Neurol Res 2015;37:624-632.
17 Barathmanikanth S, Kalishwaralal K, Sriram M, Pandian SR, Youn HS, Eom S, Gurunathan $S$ : Anti-oxidant effect of gold nanoparticles restrains hyperglycemic conditions in diabetic mice. J Nanobiotechnol 2010;8:16.

18 Zhou Z, Joslin S, Dellinger A, Ehrich M, Brooks B, Ren Q, Rodeck U, Lenk R, Kepley CL: A novel class of compounds with cutaneous wound healing properties. J Biomed Nanotechnol 2010;6:605-611.

19 Chiche J, Ricci JE, Pouyssegur J: Tumor hypoxia and metabolism - towards novel anticancer approaches. Ann Endocrinol (Paris) 2013;74: 111-114.

20 Andersson U, Wang H, Palmblad K, Aveberger $\mathrm{AC}$, Bloom O, Erlandsson-Harris $\mathrm{H}$, Janson A, Kokkola R, Zhang M, Yang H, Tracey KJ: High mobility group 1 protein (HMG-1) stimulates proinflammatory cytokine synthesis in human monocytes. J Exp Med 2000;192:565-570.

21 Tang D, Kang R, Xiao W, Zhang H, Lotze MT, Wang H, Xiao X: Quercetin prevents LPS-induced high-mobility group box 1 release and proinflammatory function. Am J Respir Cell Mol Biol 2009;41:651-660.

22 Park EJ, Yi J, Chung KH, Ryu DY, Choi J, Park $\mathrm{K}$ : Oxidative stress and apoptosis induced by titanium dioxide nanoparticles in cultured BEAS-2B cells. Toxicol Lett 2008;180:222-229.

23 Luo Y, Chihara Y, Fujimoto K, Sasahira T, Kuwada M, Fujiwara R, Fujii K, Ohmori H, Kuniyasu H: High mobility group box 1 released from necrotic cells enhances regrowth and metastasis of cancer cells that have survived chemotherapy. Eur J Cancer 2013;49:741-751. 